\title{
Criminologie
}

\section{La carrière criminelle : définition et prédiction}

\section{Marc LeBlanc}

Volume 19, numéro 2, 1986

La prédiction de la carrière criminelle

URI : https://id.erudit.org/iderudit/017241ar

DOI : https://doi.org/10.7202/017241ar

Aller au sommaire du numéro

Éditeur(s)

Les Presses de l'Université de Montréal

ISSN

0316-0041 (imprimé)

1492-1367 (numérique)

Découvrir la revue

Citer cet article

LeBlanc, M. (1986). La carrière criminelle : définition et prédiction

Criminologie, 19(2), 79-99. https://doi.org/10.7202/017241ar

\section{Résumé de l'article}

The concept of a criminal "career» is being used more and more in the recent criminological literature. This article analyzes the pertinence of explaining this concept by its specific components. To do this, the criminal activities of a sample of adolescents and a group of wards of the Montreal Court between the ages of seven and twenty-five are described (precocity, frequency, variety, gravity, aggravation, violence, duration). Two stages were detected in the criminal career, their degree of stability and paths of development. The dynamics of the criminal activity are described. Finally, the predictability of an intensification of criminal activities is analyzed. Given the high degree of stability, predictability and mobility (marked by aggravation of the criminal activity), we conclude that recourse to the concept of a criminal career is essential to a better understanding and more accurate diagnosis of the process of acting out. 


\section{LA CARRIERE CRIMINELLE : DÉFINITION ET PRÉDICTION ${ }^{1}$ Marc LeBlanc*}

The concept of a criminal "career" is being used more and more in the recent criminological literature. This article analyzes the pertinence of explaining this concept by its specific components. To do this, the criminal activities of a sample of adolescents and a group of wards of the Montreal Court between the ages of seven and twenty-five are described (precocity, frequency, variety, gravity, aggravation, violence, duration). Two stages were detected in the criminal career, their degree of stability and paths of development. The dynamics of the criminal activity are described. Finally, the predictability of an intensification of criminal activities is analyzed. Given the high degree of stability, predictability and mobility (marked by aggravation of the criminal activity), we conclude that recourse to the concept of a criminal career is essential to a better understanding and more accurate diagnosis of the process of acting out.

\section{INTRODUCTION}

Le sens du mot carrière, c'est celui d'une activité humaine qui présente des étapes, une progression. Les sociologues ne délimitent pas autrement la notion de carrière. Ainsi, Becker (1970) la définit comme le patron des ajustements séquentiels que l'individu accomplit au réseau institutionnel, les organisations formelles et informelles qui constituent l'environnement immédiat dans lequel l'occupation s'insère. D'un point de vue criminologique, il faudrait dire que la carrière criminelle est l'ajustement continu de l'individu au monde conventionnel et criminel et au système de justice dans lequel l'activité criminelle s'insère. Becker ajoute que cette série d'adaptations doit être envisagée en termes de mouvements ascendants et descendants entre diverses positions sur le continuum de carrière.

1. Cette recherche a été réalisée grâce à des contributions de la Fondation Richelieu et du ministère du Solticiteur général du Canada.

* Professeur à l’École de criminologie et chercheur associé au Centre international de criminologie comparée, Université de Montréal. 
Cette notion de carrière est relativement absente des écrits criminologiques : des histoires de cas de criminels ont été écrites (Shaw, 1931; Sutherland, 1937...); les biographies et autobiographies de criminels sont innombrables; les travaux dans la perspective du rôle délinquant sont plutôt rares (Clinard et Quinney, 1967; Gibbons, 1955; Lemert, 1975; Schur, 1971); et plus récemment les chercheurs se sont intéressés à l'identification des délinquants chroniques (Wolfgang et al., 1978; Farrington, 1981, 1984, 1985a, 1985b; West et Farrington, 1977; Peterson et al., 1980; Blumstein et al., 1980, 1985; McCord, 1981; Polk, 1978; Rojeck et Erickson, 1981; Petersilia et al., 1977; Peterson et al., 1980; Chaiken et Chaiken, 1982; Wolfgang et Tracy, 1982; Hanpaneen et Jesness, 1982; Mathias et al., 1984; Dunford et Elliot, 1984). En somme, les travaux de recherche sur l'activité antisociale, comme carrière, sont rares. Une véritable criminologie de la carrière criminelle n'est qu'embryonnaire dans les travaux les plus récents (Blumstein et al., 1982, 1985; Bellot et al., 1984; Bellot et Le Blanc, 1984).

Cette criminologie de la carrière criminelle requiert la vérification de l'existence d'une progression à travers des stades dans l'activité criminelle des individus; et c'est seulement si cette condition est remplie que nous pourrons utiliser la notion de carrière criminelle. Notre système de justice suppose l'existence de stades, puisqu'il distingue les tribunaux pour mineurs et pour adultes et les politiques criminelles qui s'appliquent à chacune de ces populations (les moins de dix-huit ans et les dix huit ans et plus) ne sont pas identiques : déjudiciarisation et traitement, surtout en milieu naturel, dans le système de justice pour mineurs et rétribution et incarcération dans le système de justice pour adultes. Le sens commun distingue le délinquant juvénile et le criminel adulte et les délinquants, eux-mêmes, reconnaissent qu'être un mineur ou un adulte n'appelle pas les mêmes attitudes, ni les mêmes comportements de la part de la société et des tribunaux : on est plus sévère avec les adultes.

\section{NOTES MÉTHODOLOGIQUES}

Deux échantillons et sept construits opérationnels serviront pour les analyses rapportées dans cet article. Un échantillon représentatif de 1684 adolescents mâles de l'île de Montréal appartenant à toutes les couches sociales et catégories occupationnelles et âgés de douze à seize ans en 1974 (voir Biron et al., 
1975). Un échantillon de 1026 individus mâles qui ont été condamnés par un tribunal pour mineurs et/ou pour adultes; ces sujets avaient quinze ans en moyenne au moment de leur recrutement et ils faisaient l'objet de mesures spécifiques : probation ou placement en internat (voir Fréchette, 1981; LeBlanc, 1983; Brill et Harvie, 1978). Notons que pour les fins de l'analyse des données, nous isolerons, à l'occasion, 526 sujets ayant été reconnus délinquants à la fois par les tribunaux pour mineurs et pour adultes.

Les sept concepts opérationnels, choisis pour rendre compte des divers aspects de l'activité criminelle comme occupation, sont les suivants : la précocité, l'âge au premier délit; la fréquence, le nombre total de délits commis; la variété, le nombre de types différents de délits; la durée, le temps en années entre le début et la fin de l'activité criminelle; la gravité, le nombre de délits pondéré par la gravité légale de chaque délit (voir Fréchette, 1981); l'aggravation ou séquence, la progression de délits mineurs vers des délits de plus en plus graves (voir Fréchette et LeBlanc, 1979); et la présence de violence, le nombre de délits contre les personnes incluant les vols à main armée. Ces composantes de l'activité criminelle sont mesurées à partir de la délinquance officielle, les condamnations devant les tribunaux pour des infractions au Code criminel canadien.

Notre stratégie d'analyse fera appel à l'échantillon d'adolescents (1 684 garçons) pour établir les taux de base; par la suite, l'échantillon de délinquants ( 1026 garçons dont 526 ayant commis des délits comme mineurs et comme majeurs) servira pour tenter de mieux comprendre la dynamique de la carrière criminelle. Les procédures d'analyse seront relativement simples : distributions de fréquences, mesures de tendances centrales (médianes), tests de la médiane, corrélations de Pearson et analyses de régression multiple et de la fonction discriminante.

\section{DÉLIMITATION DE LA CARRIËRE CRIMINELLE}

La démonstration de la pertinence de la notion de carrière criminelle pour analyser l'activité délictueuse doit se faire par l'identification d'étapes dans la participation aux activités criminelles et par la démonstration de la présence d'une aggravation à travers les infractions, d'une progression dans les activités illicites des individus. 


\subsection{DES STADES CONNUS}

Les courbes de prévalence de la délinquance juvénile et de la criminalité adulte sont bien connues des criminologues depuis plusieurs décennies (voir Glueck, 1959; Hirschi et Gottfredson, 1983; Farrington, 1984; Fréchette et LeBlanc, 1986). Chez les adolescents, elles démontrent que le nombre de jeunes délinquants commence à baisser une année après la fin de la scolarité obligatoire et, chez les adultes, elles établissent que le nombre de criminels adultes actifs diminue d'une façon appréciable à partir de vingt cinq ans. Les analyses de Farrington (1984, 1985a) et de Blumstein et al. (1982) mettent bout à bout ces deux courbes : les données soutiennent qu'après l'adolescence (dixsept ans) le nombre d'adultes criminels actifs diminue régulièrement. De plus, la lecture des données rapportées par Hirschi et Gottfredson (1983) nous permet d'avancer l'idée de l'existence de variations cycliques dans la prévalence de la délinquance : on pourrait même penser que le cycle de l'activité criminelle comprend deux phases et celle de l'adolescence aurait plus d'ampleur.

Dès 1978, LeBlanc et al. (1978) ont été amenés à réfléchir sur cette question puisque des observations, sur la délinquance cachée des mêmes adolescents à deux années d'intervalle, permettaient de soutenir l'hypothèse de l'existence de variations cycliques de la prévalence de la délinquance cachée. Les données établissaient que plus l'âge augmente, plus il y a d'adolescents qui commettent des infractions, ceci jusqu'à quinze ans; par la suite il y a une baisse appréciable de la prévalence (si on considère le pourcentage des mêmes adolescents actifs deux ans plus tard) suivi d'une remontée chez les jeunes adultes, mais celle-ci n'est jamais aussi marquée que la première.

Lorsque l'on adresse cette question aux données sur la délinquance et la criminalité officielle, il convient de calculer la participation à l'activité criminelle, à savoir combien d'individus à chaque âge sont actifs parmi ceux qui manifesteront une conduite criminelle à un moment ou à l'autre de la période d'étude. La figure 1 ne laisse plus de doute sur les variations cycliques de l'activité délinquante, cycles d'intensité variable (celui de l'adolescence étant plus marqué). Ainsi, la participation progresse jusqu'à seize ans; ensuite, elle baisse très rapidement, à dix-sept et dix-huit ans, pour reprendre un peu d'ampleur, entre dix-neuf et vingt et un ans, et descendre rapidement par la suite, atteignant alors le niveau du début de l'adolescence; 
FIGURE 1

Échantillon d'adolescents : prévalence de l'activité criminelle

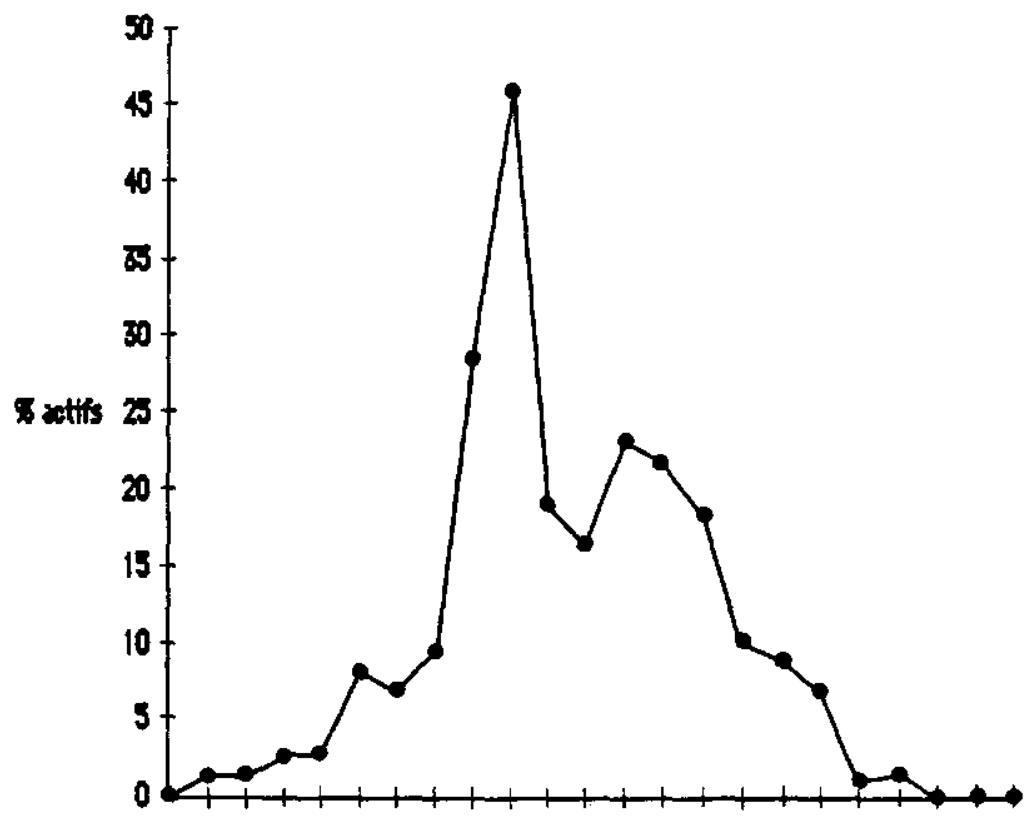

7891011121314151617181920212223242526272829

finalement, elle se stabilise, à des niveaux différents, pendant la première et la deuxième moitié de la vingtaine. Il est probable que la deuxième voussure, entre dix-neuf et vingt et un ans, s'explique par les délinquances d'occasion et de transition qui apparaissent à différents âges et généralement plus tard chez ces individus (voir tableau 2).

La figure 2 vient spécifier la nature de la participation à la carrière criminelle chez les sujets qui affichent des activités criminelles à l'adolescence et/ou à l'âge adulte. Parmi les 1026 sujets condamnés par les tribunaux, ils sont de plus en plus nombreux à participer à l'activité criminelle et, ceci, jusqu'à quinze ans, atteignant la moitié du groupe; par la suite, la participation baisse mais demeure relativement stable et élevée, pour ensuite tomber brutalement après dix-neuf ans. Parmi les 526 individus ayant été déclarés délinquants au moment de l'adolescence et reconnus coupables d'une infraction comme adultes, 


\section{FIGURE 2}

Participation dans l'activité criminelle chez les délinquants

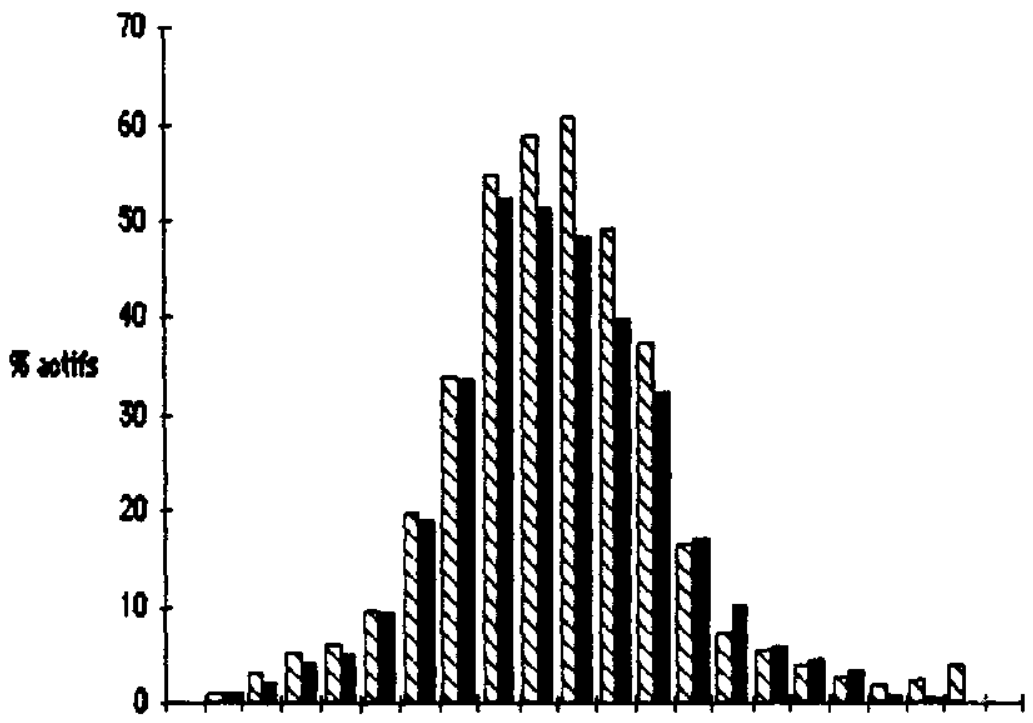

78910111213141516171819202122232423262728

Q délinquants de carrière délinquants

les mêmes tendances se font jour; toutefois, la croissance de la participation progresse jusqu'à dix-sept ans, atteignant plus de $60 \%$ du groupe, par la suite, le nombre d'individus actifs diminue mais demeure élevé jusqu'à dix-neuf ans; il chute profondément ensuite et il adopte la forme d'une courbe en $U$ au cours de la vingtaine.

Cette courbe vient confirmer que le nombre de criminels constamment productifs est restreint et que la carrière criminelle adulte est de courte durée après un départ fulgurant (voir les travaux de Blumstein et al., 1982 et les données du tableau 2). La carrière criminelle, dans sa phase la plus active, se situe donc à cheval sur l'adolescence et le début de l'âge adulte. Le creux au milieu de la vingtaine est probablement le résultat de la neutralisation qui est généralement appliquée à ces sujets, ils subissent des sentences d'emprisonnement. La reprise qui semble se dessiner au milieu de la vingtaine serait peut-être le 
point de départ d'une résurgence des activités criminelles à la sortie de prison.

En somme, la différence majeure entre un délinquant de carrière et les autres, c'est une relative constance des activités criminelles, pour la moitié d'entre eux, entre quinze et vingt ans. Il y aurait donc plusieurs stades dans la carrière délinquante : celui de l'émergence où l'activité criminelle fluctue d'intensité d'année en année et elle affecte annuellement moins de $20 \%$ des délinquants potentiels; le stade de la pleine productivité, entre quinze et dix-neuf ans, où plus de la moitié des délinquants sont actifs; et finalement, le stade de l'activité sporadique où peu de délinquants, moins de $10 \%$, sont actifs simultanément. Ces observations justifient donc l'utilisation de la notion de carrière criminelle puisque des étapes dans la participation à ce type d'activité illicite sont identifiables.

La productivité criminelle, figure 3, c'est-à-dire la moyenne de délits commis par les individus actifs à chaque âge et conduisant à une condamnation, vient confirmer ce cycle de l'activité criminelle. En effet, la moyenne annuelle est inférieure à quatre jusqu'à treize ans; elle avoisine ce niveau par la suite; et, à la fin de l'adolescence, elle se situe autour de cinq pour décroître

\section{FIGURE 3}

Nombre moyen de condamnations selon les âges

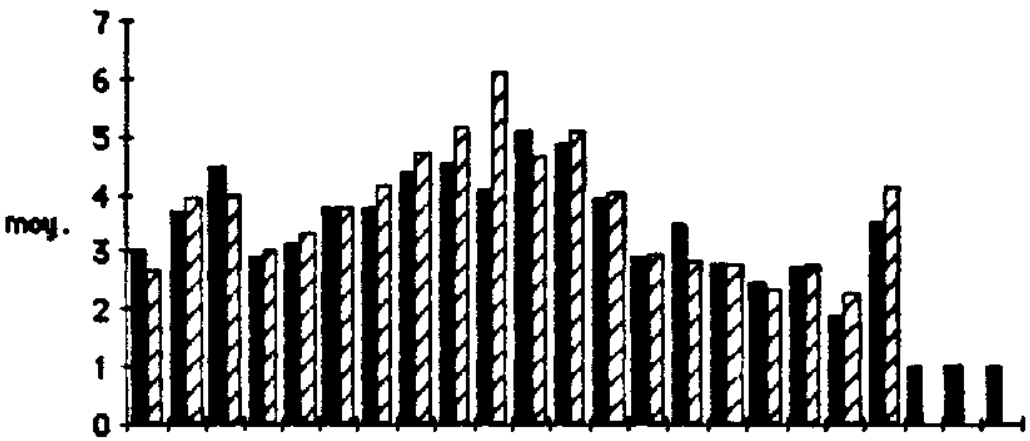

78911111111112222222222 01234567890123456789

$\square$ délinquants $\square$ carrière 
progressivement au cours de la vingtaine. Farrington et al. (1985c) rapportent une distribution semblable de la productivité criminelle, toutefois, les moyennes sont presque deux fois plus élevées que celles illustrées à la figure 3. Cette différence d'ampleur dans les moyennes s'explique probablement par des variations dans la nature des échantillons; son échantillon provient d'un quartier ouvrier tandis que le nôtre rassemble des sujets provenant de milieux socio-économiques plus diversifiés. Notons que la productivité des 526 individus, à la fois délinquants juvéniles et criminels adultes, est plus élevée que celle de l'ensemble des délinquants et ceci pour la plupart des âges à partir du milieu de l'adolescence.

\subsection{INTENSIFICATION DE L'ACTIVITÉ CRIMINELLE}

L'intensification de l'activité criminelle de la période juvénile à la phase adulte de la carrière est particulièrement visible au tableau 1. Quel que soit l'échantillon (adolescents, délinquants et délinquants de carrière) et, indépendamment de la composante de l'activité criminelle considérée (précocité, durée, volume, gravité, variété, violence), il y a presque toujours une différence remarquable entre les médianes décrivant l'activité criminelle à l'adolescence et à l'âge adulte. En somme, il est raisonnable d'utiliser la notion de carrière criminelle puisqu'il y a progression entre la délinquance juvénile et la criminalité adulte.

TABLEAU 1

Intensité de l'activité criminelle (médianes)

\begin{tabular}{|c|c|c|c|c|c|c|}
\hline & \multicolumn{2}{|c|}{$\begin{array}{l}\text { Adolescents } \\
(\mathrm{N}=1684)\end{array}$} & \multicolumn{2}{|c|}{$\begin{array}{l}\text { Délinquants } \\
(\mathrm{N}=1026)\end{array}$} & \multicolumn{2}{|c|}{$\begin{array}{c}\text { Chroniques } \\
\mathrm{N}=526 \text { ) }\end{array}$} \\
\hline & juvéniles & adultes & juvéniles & adultes & juvéniles & adultes \\
\hline $\begin{array}{l}\text { Volume } \\
\text { (n' dé.) }\end{array}$ & 1,67 & 2,63 & 5,22 & 5,02 & 6,83 & 7,25 \\
\hline $\begin{array}{l}\text { Précocité } \\
\text { (âge) }\end{array}$ & 15,2 & 19,79 & 14,12 & 18,86 & 14,05 & 18,61 \\
\hline $\begin{array}{c}\text { durée } \\
\text { (années) }\end{array}$ & 1,36 & 2,24 & 2,22 & 3 & 2,52 & 3,48 \\
\hline $\begin{array}{l}\text { gravité } \\
\text { (légale) }\end{array}$ & 3,28 & 5,78 & 13,46 & 12,27 & 17,72 & 17,64 \\
\hline variété & 1,47 & 1,42 & 2,95 & 2,65 & 3,44 & 3,36 \\
\hline $\begin{array}{l}\text { violence } \\
\text { ( } n^{\prime \prime} \text { dé.) }\end{array}$ & 1,37 & 2,06 & 1,67 & 2,24 & 1,7 & 2,56 \\
\hline
\end{tabular}


Considérant nos observations sur la présence d'étapes dans l'activité criminelle et l'existence d'une aggravation des agirs, le concept de carrière criminelle doit être employé en criminologie et il convient de l'opérationnaliser avec les sept composantes que nous avons retenues. Ces composantes structurales de l'activité criminelle ne sont pas suffisantes car il ne s'agit que de concepts descriptifs.

\section{CONFIGURATION DE LA CARRIÈRE CRIMINELLE}

Pour procéder à une analyse criminologique de l'activité criminelle comme carrière, il convient d'avoir recours à des concepts développementaux. Nous avons retenu ceux de stabilité, de profil de mobilité et de dynamique de l'activité criminelle. Voyons comment se configure la carrière criminelle des délinquants de notre échantillon.

\subsection{STABILITÉ ET MOBILITÉ DANS L'ACTIVITÉ CRIMINELLE}

La stabilité réfère à la constance dans la pratique des activités délictueuses et la mobilité se rapporte aux variations de l'intensité de la productivité criminelle à travers le temps et, plus particulièrement, entre l'adolescence et le début de l'âge adulte.

Analysons la stabilité et la mobilité dans l'activité délinquante, d'abord pour l'échantillon d'adolescents mâles. Il y a une stabilité certaine de la prévalence (présence ou absence) de l'activité criminelle au cours de l'adolescence et/ou de l'âge adulte : en effet, l'association entre elles est statistiquement significative $\left(\mathrm{X}^{2}=83, \mathrm{dl}=1, \mathrm{p} \leqslant 0,001\right)$ et la corrélation (Eta) est de 0,23. Sur le plan de la mobilité (figure 4), notons que $86 \%$ de la population ne s'engagent jamais dans une activité criminelle officielle alors que $2 \%$ poursuivent une carrière criminelle comme mineurs et comme majeurs. $9 \%$ s'engagent sur le tard dans l'activité criminelle, c'est-à-dire à partir de dixhuit ans et $2 \%$ décrochent de cette activité puisqu'ils ne manifestent des activités criminelles qu'au moment de l'adolescence.

Cette dernière proportion est assez surprenante même si le phénomène de la délinquance sur le tard est connu (Cormier $e t$ al., 1962a, 1962b); si peu d'adolescents sont condamnés par les 
FIGURE 4

Mobilité dans l'activité criminelle

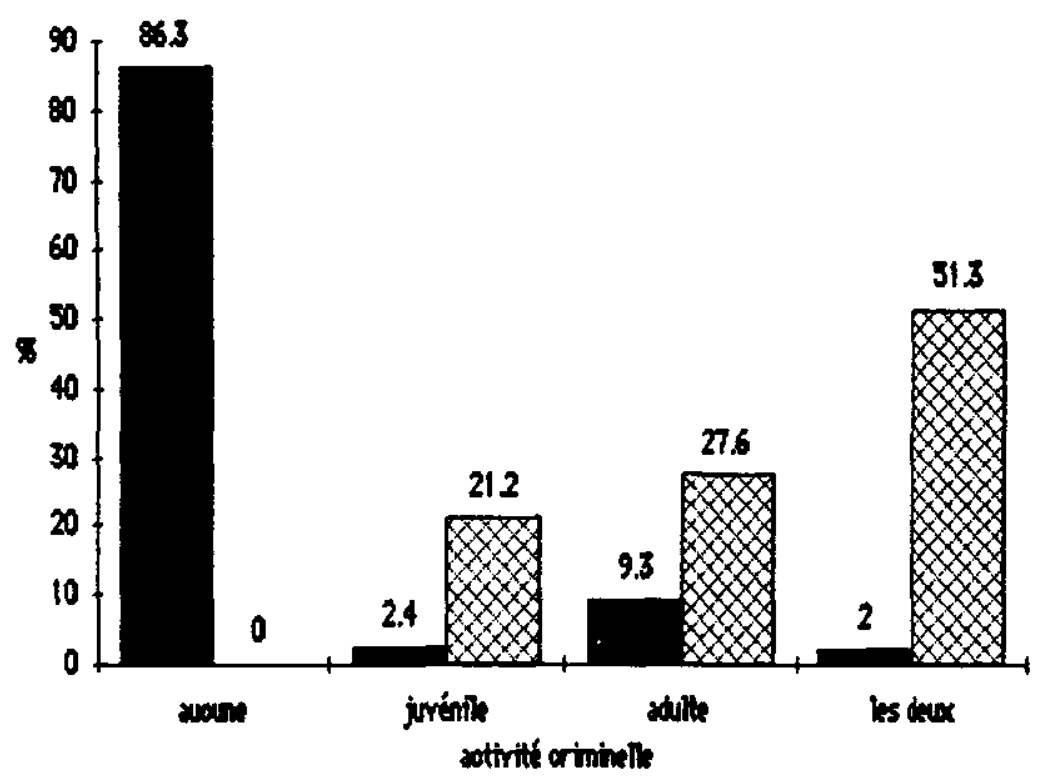

adolescents $Q$ délinquants

tribunaux pour mineurs c'est sûrement en raison de la politique d'intervention minimale ou de déjudiciarisation qui est généralisée au niveau de l'énoncé de la loi (Loi sur la protection de la jeunesse, 1977), des pratiques de la police (LeBlanc, 1978) et des procureurs de la couronne (Trépanier et Gagnon, 1984) et qui se manifeste par la présence de nombreuses décisions sans mesure spécifique de la part des tribunaux pour mineurs (LeBlanc et Beaumont, 1985).

Si nous analysons la stabilité et la mobilité dans l'activité criminelle chez les sujets ayant été condamnés au moins une fois soit comme jeunes délinquants et/ou comme criminels adultes, nous devons conclure à sa stabilité (eta $=0,32, X^{2}=103,08$, $\mathrm{dl}=1, \mathrm{p} \leqslant 0,001$; mais la répartition des recrues à l'âge adulte $(28 \%)$, des persistants $(51 \%)$ et de ceux qui se désistent avant dix-huit ans $(21 \%)$ est fort différente de celle rapportée précé- 
demment pour l'ensemble des adolescents (figure 4). Notons que Wolfgang (1974) et Shannon (1978) rapportent des proportions tout à fait comparables de recrues, de persistants et de décrocheurs dans leurs cohortes de délinquants.

Puisque l'activité délictueuse n'est que relativement stable entre l'adolescence et l'âge adulte, il convient d'analyser la mobilité dans la carrière criminelle, comme passage de la délinquance juvénile à la criminalité adulte. Pour ce faire, il est nécessaire de classer les 1026 individus ayant commis des infractions comme mineurs et/ou comme adultes en sept groupes, la figure 5 schématise cette distribution : ceux qui ne commettent qu'un seul délit, $16 \%$; ceux qui arrêtent après une délinquance juvénile parfois intense, $14 \%$; ceux qui ne commettent qu'un seul délit, $16 \%$; ceux qui arrêtent après une délinquance juvénile parfois intense, $14 \%$; ceux qui ne comdébutent leurs activités délictueuses à l'âge adulte, les délinquants sur le tard, $14 \%$; ceux dont l'activité est stable et persis-

FIGURE 5

Mobilité dans la carrière criminelle

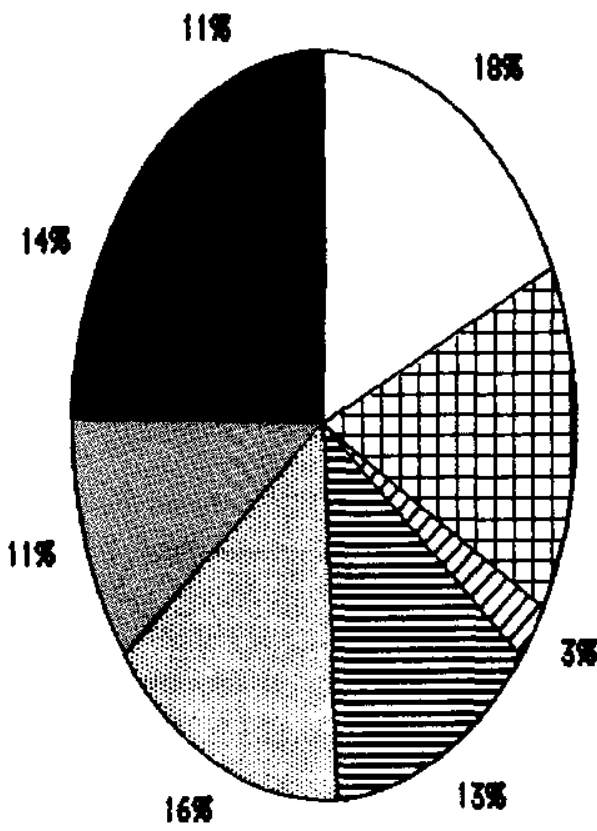

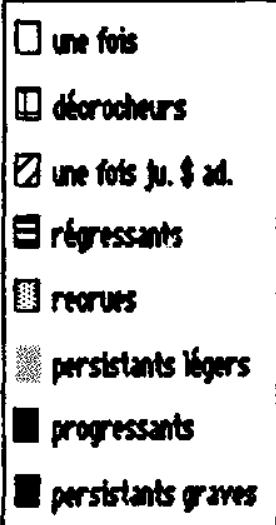


TABLEAU 2

Caractéristiques de l'activité criminelle selon le type de mobilité dans la carrière (médianes)

\begin{tabular}{|c|c|c|c|c|c|c|c|c|c|}
\hline & une fois & décro. & une $\mathrm{j}$ et a & recrues & regress. & stab. lé. & progress. & chroniq. & test méd. \\
\hline $\begin{array}{l}\text { J. volume } \\
\text { (no dé.) }\end{array}$ & 1 & 4,39 & 1 & - & 12,27 & 4,57 & 2,75 & 18,8 & 0,001 \\
\hline J. précocité & 15,21 & 13,96 & 15,56 & - & 13,59 & 14,14 & 14,74 & 13,26 & 0,001 \\
\hline $\begin{array}{l}\text { J. durée } \\
\text { (années) }\end{array}$ & 1 & 2,13 & 1 & - & 3,27 & 2,19 & 1,5 & 3,97 & 0,001 \\
\hline $\begin{array}{c}\text { J. gravité } \\
\text { (légale) }\end{array}$ & 2,31 & 11,67 & 2,3 & - & 32,1 & 11,67 & 6,07 & 55 & 0,001 \\
\hline $\begin{array}{l}\text { J. variété } \\
\text { (n }{ }^{0} \text { types) }\end{array}$ & 1 & 2,66 & 1,13 & - & 4,24 & 2,98 & 2 & 5,69 & 0,001 \\
\hline $\begin{array}{l}\text { J. violence } \\
\left(\mathrm{n}^{0} \text { dé.) }\right.\end{array}$ & 1 & 1,96 & 1 & - & 1,73 & 1,28 & 1,24 & 3,8 & 0,001 \\
\hline $\begin{array}{c}\text { A. volume } \\
\text { (n'ó. dé.) }\end{array}$ & 1,87 & 一 & 1 & 5,21 & 2,73 & 5,04 & 12,03 & 16,33 & 0,001 \\
\hline $\begin{array}{l}\text { A. précocité } \\
\text { (années) }\end{array}$ & 20,44 & - & 19,41 & 18,95 & 18,89 & 18,85 & 18,7 & 18 & 0,001 \\
\hline $\begin{array}{l}\text { A. durée } \\
\text { (années) }\end{array}$ & 1,89 & - & 1 & 3,1 & 2,45 & 3,14 & 4,66 & 4,62 & 0,001 \\
\hline $\begin{array}{c}\text { A. gravité } \\
\text { (légale) }\end{array}$ & 3,14 & - & 4,28 & 12,21 & 7,7 & 12,09 & 29,39 & 45,67 & 0,001 \\
\hline $\begin{array}{l}\text { A. variété } \\
\text { (n'types) }\end{array}$ & 1 & - & 1,06 & 2,62 & 1,74 & 2,66 & 4,8 & 5,52 & 0,001 \\
\hline $\begin{array}{l}\text { A. violence } \\
\text { (n' dé.) }\end{array}$ & 1,16 & - & 1,7 & 2,27 & 2,17 & 2,27 & 2,34 & 5,7 & 0,001 \\
\hline
\end{tabular}


tante, entre deux et neuf délits comme mineurs et comme adultes, $10 \%$; ceux qui progressent vers une activité plus intensive, $16 \%$ et finalement, ceux dont l'activité délictueuse demeure élevée et stable (dix délits ou plus comme adolescents et comme adultes), $10 \%$.

Le tableau 2 démontre que ces types de mobilité signifient une configuration particulière d'activités criminelles; les tests de la médiane, tous statistiquement significatifs, viennent confirmer cette observation. Ainsi, la délinquance d'une occasion, juvénile et/ou adulte, est tardive et peu grave. Ceux qui décrochent avaient la troisième plus intensive délinquance juvénile et ceux qui diminuent leur activité criminelle se remarquaient par une productivité juvénile au deuxième rang. La progression de ceux qui accélèrent leurs activités délictueuses est tout à fait évidente et il en est de même pour la carrière persistante mineure et majeure.

\subsection{LA DYNAMIQUE DE L'ACTIVITÉ CRIMINELLE}

Le tableau 3 rapporte les corrélations entre les composantes de l'activité criminelle juvénile et adulte; il renseigne sur la dynamique de la conduite délinquante officielle et il illustre, avec la figure 6, l'articulation des composantes structurales de l'activité criminelle. Cette figure montre que le pivot de la conduite est formé de la précocité, de la durée et de la variété; elles s'enchaînent pour constituer le moteur de l'activité délictueuse; plus elles s'amplifient plus elles supportent un volume élevé; celui-ci, par voie de conséquence, les aidera à produire une activité de plus en plus grave et composée de délits de violence.

La figure 6 laisse aussi voir que la dynamique de la conduite criminelle adulte est sensiblement différente de celle que nous avons décrite pour l'activité criminelle juvénile. La séquence, la durée et la variété apparaissent les résultantes de la précocité et, en contrepartie, elles supportent un volume élevé; la commission de délits de violence ressort, ici aussi, comme l'aboutissement final de la productivité criminelle mais cette fois à travers le volume.

L'analyse de la transformation d'une délinquance juvénile significative en une criminalité adulte importante révèle que celle-ci semble s'exprimer à travers trois mécanismes complémentaires (figure 6) : le volume de l'activité juvénile conditionne 


\section{TABLEAU 3}

Corrélations de Pearson entre les composantes de l'activité criminelle ( $N: 1026)$

\begin{tabular}{|c|c|c|c|c|c|c|c|c|c|c|c|c|}
\hline & $\mathbf{J}:$ préc. & $\mathrm{J}$ : séq. & $\mathrm{J}:$ dur. & $\mathrm{J}$ : var. & $\mathrm{J}:$ vio. & J : vol. & A : préc. & A : séq. & A : dur. & A : var. & A : vio. & A : vol. \\
\hline $\begin{array}{l}\mathrm{J}: \text { précocité } \\
\mathrm{J}: \text { séquence } \\
\mathrm{J}: \text { durée } \\
\mathrm{J}: \text { variété } \\
\mathrm{J}: \text { violence } \\
\mathrm{A}: \text { précocité } \\
\mathrm{A}: \text { séquence } \\
\mathrm{A}: \text { durée } \\
\mathrm{A}: \text { variété } \\
\mathrm{A}: \text { violence } \\
\mathrm{A}: \text { volume }\end{array}$ & & $-0,11$ & $\begin{array}{r}-0,63 \\
0,42\end{array}$ & $\begin{array}{c}-0,33 \\
0,5 \\
0,71\end{array}$ & $\begin{array}{r}-0,11 \\
0,19 \\
0,26 \\
0,46\end{array}$ & $\begin{array}{r}-0,31 \\
0,33 \\
0,63 \\
0,68 \\
0,61\end{array}$ & $\begin{array}{l}-0,05 \\
-0,24 \\
-0,25 \\
-0,29 \\
-0,24\end{array}$ & $\begin{array}{r}-0,05 \\
0,11 \\
0,13 \\
0,09 \\
0,02 \\
-0,18\end{array}$ & $\begin{array}{r}-0,07 \\
0,1 \\
0,21 \\
0,16 \\
-0,02 \\
-0,04 \\
0,43\end{array}$ & $\begin{array}{r}-0,05 \\
0,16 \\
0,22 \\
0,25 \\
0,12 \\
-0,41 \\
0,44 \\
0,77\end{array}$ & $\begin{array}{c}-0,01 \\
0,16 \\
0,15 \\
0,3 \\
0,6 \\
-0,18 \\
0,01 \\
0,01 \\
0,18\end{array}$ & $\begin{array}{r}-0,11 \\
0,99 \\
0,25 \\
0,2 \\
0,27 \\
-0,33 \\
0,29 \\
0,57 \\
0,67 \\
0,44\end{array}$ \\
\hline
\end{tabular}


FIGURE 6

La dynamique de l'activité criminelle juvénile

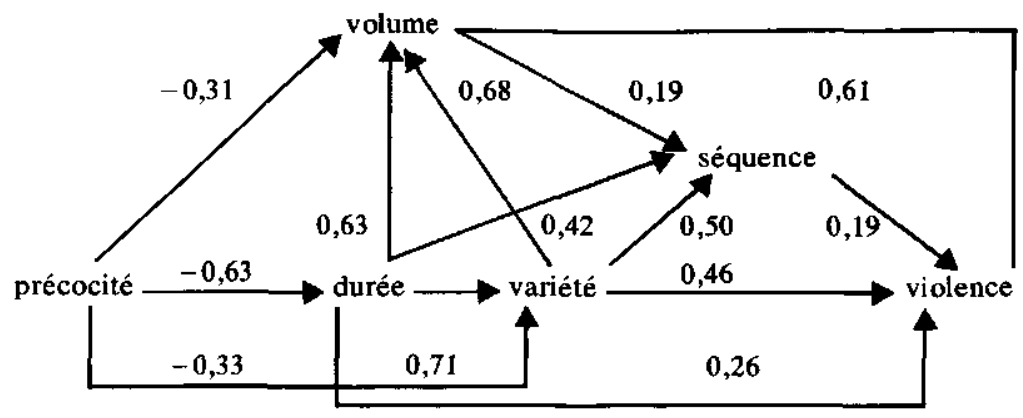

Passage de la délinquance juvénile à la criminalité adulte

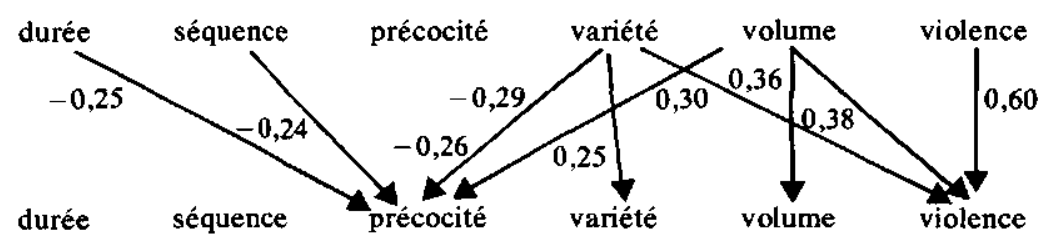

La dynamique de l'activité criminelle adulte

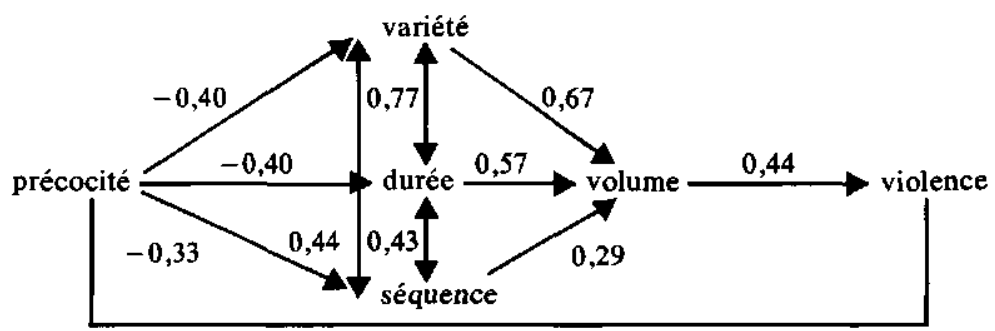

le niveau de la productivité adulte (les corrélations sont fortes entre le volume juvénile et des composantes de l'activité criminelle adulte : violence et précocité); la violence adolescente est un déterminant puissant de la violence adulte (corrélation de 0,60 entre la présence de délits de violence durant l'adolescence et au cours de l'âge adulte); et, principalement, l'ampleur de l'activité criminelle juvénile (durée, séquence, variété et volume) affecte la précocité adulte qui elle, en retour, déclenche l'activité criminelle adulte. 
Ces données sur la dynamique de l'activité criminelle ne constituent pas une analyse définitive de la question de la dynamique de l'activité criminelle, il conviendra de recourir à des techniques statistiques plus complexes, ceci pour mieux comprendre le développement de la carrière criminelle.

\section{LA CRIMINALITÉ ADULTE EST PRÉVISIBLE}

Non seulement y a-t-il une corrélation significative entre le volume de l'activité criminelle juvénile et le volume de l'activité criminelle adulte chez les sujets délinquants étudiés, mais celle-ci est prévisible à partir des composantes de celle-là : la corrélation multiple entre elles est de 0,39 ( $15 \%$ de la variance de l'ampleur de l'activité criminelle adulte est ainsi expliquée par les composantes de l'activité criminelle juvénile pour l'échantillon de délinquants). Les prédicteurs sont par ordre d'importance : la précocité $(R=0,11)$, l'aggravation $(R=0,14)$, la violence $(R=0,29)$, la variété $(R=0,29)$, le volume $(R=0,38)$ et la durée $(R=0,39)$.

Cette conclusion est encore plus probante en ce qui concerne l'activité criminelle de violence à l'âge adulte : la corrélation multiple est de 0,61 (37\% de la variance est expliquée par les composantes de l'activité criminelle juvénile) et l'ordre d'importance des prédicteurs juvéniles est le suivant : précocité $(R=0,02)$, l'aggravation $(R=0,16)$, violence $(R=0,60)$, variété $(R=0,606)$, volume $(R=0,606)$ et durée $(R=0,607)$.

Si l'activité criminelle est prévisible et si la carrière se définit principalement par le passage de la délinquance juvénile à la criminalité adulte, il convient de se demander s'il est possible de prédire adéquatement ce passage, ceci en n'utilisant que des informations sur l'activité criminelle juvénile. L'application de l'analyse de la fonction discriminante (Lambda $=0,85$, $\mathrm{X}^{2}=162,71, \mathrm{dl}=4, \mathrm{p} \leqslant 0,001$, corrélation canonique de 0,38 ) permet de définir une fonction composée du volume (coefficient standardisé discriminant $=2,62)$, de la variété $(-1,38)$, de l'aggravation $(0,38)$ et de la violence $(-0,40)$ qui classifie avec $65,79 \%$ de succès ceux qui consolideront une carrière amorcée à l'adolescence de ceux qui décrocheront avant l'âge adulte.

De plus, l'ampleur et la nature de celle-ci est aussi prévisible puisque l'analyse de la fonction discriminante produit des statistiques de qualité $($ Lambda $=0,91$; corrélation canonique $=$ 
0,$\left.30 ; X^{2}=77,43, d l=4, p \leqslant 0,001\right)$ pour distinguer ceux qui commettront d'une à neuf infractions criminelles par rapport à ceux qui en feront dix et plus. Cette fonction discriminante utilise les indices suivants de délinquance juvénile : aggravation $(-0,49)$, variété $(3,49)$, volume $(-3,04)$ et violence $(0,16)$. Son efficacité n'est pas négligeable avec $61,56 \%$ de bonnes classifications. Ce résultat sous-tend que les sujets qui diminuent leur activité criminelle à l'âge adulte ne sont pas prévisibles ( $23 \%$ de bonnes classifications), tandis que ceux qui l'augmentent le sont à $59 \%$ et que ceux qui continuent au même rythme le sont à $97 \%$ pour une carrière d'une intensité moyenne et à $86 \%$ si elle est d'intensité forte.

En ce qui concerne la violence adulte, les résultats sont encore plus probants : Lambda $=0,92$; corrélation canonique $=$ 0,$29 ; X^{2}=84,76, d l=2 ; p \leqslant 0,001$. Ainsi, il en résulte $69,2 \%$ de bonnes classifications en utilisant deux prédicteurs : la violence juvénile $(0,82)$ et l'aggravation $(-0,28)$.

En somme, compte tenu du degré de stabilité et de prévisibilité de l'activité criminelle et de la mobilité marquée par l'aggravation de celle-ci, le recours au concept de carrière criminelle apparaît essentiel. Cette carrière se manifeste, chez un nombre substantiel de sujets, principalement par le passage d'une activité criminelle comme mineurs et comme majeurs. Une des questions essentielles à laquelle il faudra répondre dans nos travaux ultérieurs se formule ainsi : qu'est-ce qui décrochent, sur les plans social, psychosocial et psychologique, ceux qui se désistent après une délinquance juvénile remarquable, de ceux qui consolident une carrière criminelle, de ceux qui entreprennent des activités criminelles sur le tard?

\section{CONCLUSION}

La pertinence du concept de carrière criminelle, pour analyser l'activité criminelle des individus, a été soutenue par la démonstration de l'existence d'étapes dans l'activité criminelle et par l'identification d'une intensification de sa gravité d'un stade à l'autre, de l'adolescence à l'âge adulte. Par la suite, nous avons débroussaillé ce champ par l'étude de la stabilité, de la mobilité et de la dynamique de l'activité criminelle. Le résultat de ces analyses se résume dans l'existence d'une activité criminelle d'occasion (une condamnation comme mineur et/ou adulte); 
d'une activité criminelle de transition (limitée à l'adolescence ou à l'âge adulte); et de quelques formes de carrières criminelles d'intensité variable.

S'il apparaît essentiel de raffiner les procédures d'analyse de la carrière criminelle, il nous apparaît plus important de faire déborder notre concept opérationnel de carrière criminelle sur l'ensemble du style de vie de l'individu. Et, ainsi, de chercher à comprendre pourquoi il y a des creux dans l'activité criminelle, pourquoi il y a des phases d'accalmie qui ne s'expliquent pas par l'emprisonnement. En effet, il nous apparaît utile de mettre en parallèle l'activité criminelle avec les activités de travail conventionnel, les périodes d'inactivité complète, les moments de dépendance économique (réception d'aide sociale, d'assurance chômage,...), ... avec toute l'occupation du temps de ces individus. Une telle analyse, si elle distingue des configurations spécifiques d'activités en regard des types de mobilité dans la carrière criminelle, nous permettra de construire un portrait compréhensif de la carrière criminelle.

\section{RÉFÉRENCES}

BECKER, H.S. (1970), Sociological Work: Method and Substance, Chicago, Aldine.

BELLOT, S., M. LEBLANC (1984), le Vol à main armée parmi l'activité délinquante, Montréal, Centre international de criminologie comparée, Université de Montréal.

BELLOT, S., M. DIONNE, P. PINSONNEAULT (1984), le Vol à main armée par ses auteurs, Montréal, Centre international de criminologie comparée, Université de Montréal.

BIRON, L., A. CAPLAN, M. LEBLANC (1975), la Construction de l'échantillon, la cueillette des données et leur préparation, Montréal, Groupe de recherche sur l'inadaptation juvénile, Université de Montréal.

BLUMSTEIN, A., S. MOITRA (1980), "The Identification of "Career Criminals» from "Chronic Offenders" in a Cohort», Law and Policy Quarterly, $2: 321-334$.

BLUMSTEIN, A., J. COHEN, P. HSIEH (1982), The Duration of Adult Criminal Career, Washington, the National Institute of Justice.

BLUMSTEIN, A., D.P. FARRINGTON, S. MOITRA (1985), «Delinquency careers : innocents, desisters and persisters", dans M. Tomry, N. Morris, Crime and Justice, vol. 6, Chicago, University of Chicago Press. 
BRILL, R., E. HARVIE (1978), Client Characteristics, Final Report $n^{\circ} 3$, Montréal, Groupe de recherche sur l'inadaptation juvénile, Université de Montréal.

CHAIKEN, J., M.R. CHAIKEN (1982), Varieties of Criminal Behavior, Santa Monica, Rand Corporation.

CLINARD, M.B., R. QUINNEY (1967), Criminal Behavior Systems : a Typology, New York, Holt, Rinehart \& Winston.

CORMIER, B., M. KENNEDY, L. SANGOWITCZ, M. TROTTIER (1962a), «The Natural History of Criminality and Some Tentative Hypothesis on its Abatment», Canadian Journal of Criminology, 1, $4: 35-49$.

CORMIER, B., M. KENNEDY, L. SANGOWITCZ, M. TROTTIER (1962a), «Presentation of a Basic Classification for Clinical Work and Research in Criminology", Canadian Journal of Criminology, 1, $4: 21-34$.

DUNFORD, F.N., D.S. ELLIOT (1984), «Identifying Career Offenders Using Self-Reported Data", Journal of Research in Crime and Delinquency, $21,1: 57-86$.

FARRINGTON, D.P. (1981), Delinquency from 10 to 25. Society for Life and History Research, Monterey, California.

FARRINGTON, D.P. (1984), Age, Crime and Longitudinal Research, 1984 Annual Meeting of the American Society of Criminology.

FARRINGTON, D.P. (1985a), «Stepping Stones to Adult Criminal Careers", dans D. Olweys, M.R. Yarrow, J. Block (édit.), Development of Antisocial and Prosocial Behavior, New York, Academic Press.

FARRINGTON, D.P. (1985b), Early Precursors of High Rate Offending, Paper presented at the executive session on delinquency and the family, Harvard University.

FARRINGTON, D.P., B. GALLAGER, L. MORLEY, R.J. ST-LEDGER, D.J. WEST (1985c), Cambridge Study in Delinquent Development : Long Term Follow-Up, First Annual Report, Institute of Criminology, University of Cambridge.

FRÉCHETTE, M. (1981), Portrait de la délinquance, Montréal, Groupe de recherche sur l'inadaptation juvénile, Université de Montréal.

FRÉCHETTE, M., M. LEBLANC (1979), la Délinquance cachée à l'adolescence, Cahier 1, Inadaptation juvénile, Montréal, Groupe de recherche sur l'inadaptation juvénile, Université de Montréal.

FRÉCHETTE, M., M. LEBLANC (1986), Des délinquances : émergence et développement, Chicoutimi, Gaétan Morin.

GIBBONS, D.C. (1955), Changing the Lawbreakers, Englewood Cliffs, Prentice Hall.

GLUECK, S., E. GLUECK (1959), Predicting Delinquency and Crime, Cambridge, Harvard University Press.

HAMPERIAN, D.M., R. SCHUSTER, S. DINITZ, J.P. CONRAD (1978), The Violent Few, Lexington, Heath.

HAAPANEN, R.A., C.F. JERSNESS (1982), Early Identification of the Chronic Offender, Sacramento, California Youth Authority. 
JESNESS, C. (1981), Delinquency in a Sacramento Birth Cohort, Sacramento, California Youth Authority.

HIRSCHI, T., M. GOTTFREDSON (1983), "Age and the Explanation of Crime", American Journal of Sociology, 89, 3 : 552-584.

LEBLANC, M. (1978), «Police Diversion of Juveniles in the 60's and the 70's : a New or an Old Phenomenon", Crime and Justice, 6,3 : 165-169.

LEBLANC, M., L. BIRON, G. CÔTÉ, L. PRONOVOST (1978), "La délinquance juvénile : son développement en regard du développement psychosocial durant l'adolescence», Annales de Vaucresson, 15:11-65.

LEBLANC, M. (1983), Boscoville : la rééducation évaluée, Montréal, $\mathrm{HMH.}$

LEBLANC, M., H. BEAUMONT (1985), Description du fonctionnement du Tribunal de la jeunesse de Montréal entre mai 1981 et avril 1982, Montréal, Cérij.

LEMERT, E.M. (1975), «Primary and Secondary Deviance», dans S.H. Traub, C.B. Little (édit.), Theories of Deviance, Itasca, Peacock.

LOI SURLA PROTECTION DE LA JEUNESSE. L.Q. 1977, c. 20.

MATHIAS, R.A., P. DEMURO, R.S. ALLINSON (1984), Violent Juvenile Offenders : an Anthology, Washington, National Council on Crime and Delinquency.

MC CORD, J. (1981), "A Longitudinal Perspective on Patters of Crime», Criminology, 19, 211-218.

PETERSILIA, J.R., P.W. GREENWOOD, M.M. LAVIN (1977), Criminal Careers ou Habitual Criminals, Santa Monica, Rand Corporation.

PETERSON, M., H. BRIAKER, S. POLICH (1980), Doing Crime : a Survey of California Inmates, Santa Monica, Rand Corporation.

POLK, K. (1978), Teenage Delinquency in Small Town America, Washington, National Institute of Mental Health.

ROJECK, D.G., M.L. ERICKSON (1982), "Delinquency Careers», Criminology, $20: 5-28$.

SCHUR, E.M. (1971), Labeling Deviant Behavior : its Sociological Implications, New York, Harper \& Row.

SHANNON, L.W. (1978), Assessing the Relationship of Adult Criminal Careers to Juvenile Careers, Iowa, Urban Community Research Center.

SHANNON, L.W. (1981), Assessing the Relationship of Adult Criminal Careers to Juvenile Delinquency : a Study of Three Birth Cohorts, Washington, National Institute of Juvenile Justice and Delinquancy Prevention.

SHAW, C.R. (1931), The Natural History of a Delinquent Career, Chicago, University of Chicago Press.

SUTHERLAND, E.H. (1937), The Professional Thief, Chicago, University of Chicago Press.

TRÉPANIER, J., R. GAGNON (1984), la Déjudiciarisation à la cour du Bienêtre social de Montréal, Montréal, École de criminologie, Université de Montréal. 
WEST, D.J., D.P. FARRINGTON (1977), The Delinquent Way of Life, New York, Crane Russak.

WOLFGANG, M.E. (1974), "Crime in a Birth Cohort», dans R. Hood (édit.), Crime, Criminology and Public Policy: Essays in Honour of Sir Leon Radjinowicz, London, Heinemann.

WOLFGANG, M.E., R.M. FIGLIO, T.E. SELLIN (1972), Delinquency in a Birth Cohort, Chicago, University of Chicago Press.

WOLFGANG, M.E., P.E. TRACY (1982), The 1945 and the 1958 Birth Cohorts : a Comparison of the Prevalence, Incidence and Severity of Delinquent Behavior, Paper presented at the Conference on Public danger, Dangerous Offenders and the Criminal Justice System. 